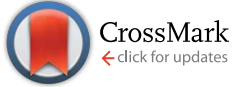

Cite this: RSC Adv., 2017, 7, 137

Received 11th November 2016 Accepted 26th November 2016

DOI: 10.1039/c6ra26677k

www.rsc.org/advances

\section{Large-sized sandpaper coated with solution- processed aluminum for a triboelectric nanogenerator with reliable durability $\dagger$}

\begin{abstract}
Daewon Kim, ${ }^{a}$ Hye Moon Lee ${ }^{b}$ and Yang-Kyu Choi ${ }^{a}$
Ambient mechanical energy, which is abundant in the living environment, is a promising energy harvesting source. This technology can provide an eco-friendly and sustainable form of energy. Here, a novel solutionprocessed aluminum coating (SC) method to create an electrode, consisting of sandpaper, is demonstrated for a triboelectric nanogenerator (TENG). The proposed SC method harnesses the strong adhesion of Al to the target substrate, with conformal deposition of Al following a pre-existing nano-to-micro surface morphology, and offering good thickness controllability without discontinuities, and the ability to coat an Al film in a manner unrestricted by the size or type of substrate. The fabricated TENG using the SC method is capable of serving as an energy supplier and can be commercialized for the Internet of Things (loT) due to its high output power and low-cost.
\end{abstract}

\section{Introduction}

At present, the potential to harvest mechanical energy from the ambient environment is attracting interest due to the ubiquitous nature and abundance of such a source. ${ }^{1}$ Thus far, various energy harvesting technologies for scavenging mechanical energy have been developed based on piezoelectrics, ${ }^{2-4}$ electromagnetics, ${ }^{5-7}$ and electrostatics. ${ }^{8-10}$ As a new energy harvesting technology, a recently invented triboelectric nanogenerator (TENG) has provided an effective approach to convert mechanical energy into electrical energy, based on the conjugation of two physical phenomena, triboelectrification (also known as contact-electrification) and electrostatic induction. ${ }^{11-16}$

Many studies have reported enhancements of the output power of the TENG for a self-powered devices in the Internet of Things (IoT) era. ${ }^{17-19}$ In order to improve the output power, the surface charge density induced by the triboelectrification process, closely related to the effective contact area, should be increased as much as possible. The effective contact surface area can be enlarged with various micro- and/or nano-scaled

${ }^{a}$ School of Electrical Engineering, Korea Advanced Institute of Science and Technology (KAIST), 291 Daehak-ro, Yuseong-gu, Daejeon 34141, Republic of Korea

${ }^{b}$ Powder \& Ceramics Division, Advanced Characterization and Analysis Group, Korea Institute of Materials Science (KIMS), 797 Changwondaero, Seongsan-gu, Changwon 51508, Republic of Korea

† Electronic supplementary information (ESI) available: Photograph of nucleation of aluminum growth on arbitrary substrate nanostructured and various substrates coated by solution-processed aluminum, the effect of contact electrode fabricated by solution processed aluminum coating on output performance, switching test, movie clip including that the real-time green 120 LEDs are lit up with serial connection by the fabricated TENG. See DOI: 10.1039/c6ra26677k surface engineering approaches, such as lithography, a plasma treatment, etching, and casting..$^{\mathbf{2 0 - 2 5}}$

Sandpaper is typically used to make a surface rougher or smoother as well as to remove a layered material. Sandpaper is classified according to the grit number, which represents the number of holes per linear inch in a sieve screen. A larger grit number implies a finer abrasive surface. Sandpaper is inexpensive, flexible, durable and widely available. Moreover, it inherently harnesses nano-to-micro scale roughness levels. Compared to the conventional substrates for the TENG, it does not need additional expensive and time-consuming fabrication processes to achieve nano-to-micro roughness to thus enhance the effective contact area of the TENG.

During the operation of a triboelectric nanogenerator, it requires one or two electrodes to enable the current to flow. Any metallic substance can be used for an electrode; ${ }^{26-28}$ however, aluminum (Al) is one of the most appropriate metals due to its high electrical conductivity and ductility and its low cost. For these reasons, $\mathrm{Al}$ has commonly been used as an electrode for a TENG. ${ }^{29-32}$

Recently, a novel method of depositing thin-film aluminum, formed by a solution-process on various substrates, has been investigated. ${ }^{33}$ The method is astonishingly simple and minimizes the defect density. Given that a general solutionprocessed Al coating fails to preclude the extremely active reactions of $\mathrm{Al}$ with oxygen and moisture, the coating process is generally conducted by a vacuum deposition process. However, vacuum deposition is linked to a few drawbacks. A large-sized substrate demands a large volume, which inevitably increases the cost and lowers the throughput. On the other hand, the solution-processed coating (SC) method suppresses the generation of a thin aluminum oxide layer $\left(\mathrm{Al}_{2} \mathrm{O}_{3}\right)$ by directly 
decomposing the Al precursor on a target surface. Furthermore, the SC method allows the outstanding adhesion of $\mathrm{Al}$ onto nearly all solid substrates and conformal deposition, creating a uniform thickness film which is equal in thickness everywhere along the interface. This method also offers very thin film deposition without any discontinuities or cracks.

In this work, a novel SC method was utilized for the formation of a metal electrode in a triboelectric nanogenerator which operates in the contact-separation mode. A SC method for a sandpaper TENG that harnesses a few distinctive merits, i.e., the conservation of the pre-existing surface morphology of the substrate as it is due to its good conformality, strong adhesion of Al to the target substrate, film deposition with high controllability without discontinuities or cracks, and versatility not restricted by the size or type of substrate, is demonstrated. It is also confirmed that the sandpaper TENG exhibits mechanically and electrically excellent durability against mechanical friction and adhesion. Further, this SC method can be used to coat $\mathrm{Al}$ onto various substrates with a large area, making it readily available as an energy supply which can be commercialized.

\section{Results and discussion}

Fig. 1a depicts a schematic illustration of the two-step fabrication procedure of the solution-processed aluminum coating (SC) method. This SC method can be applied to any substrate, even those with rough morphologies, due to the fact that the method is based essentially on a solution process. A catalytic pretreatment of a dried substrate was conducted to decrease the activation energy of the decomposition process, as the decomposition of $\mathrm{AlH}_{3}$ into $\mathrm{Al}$ and $\mathrm{H}_{2}$ occurs at about $165^{\circ} \mathrm{C}$ (see the detailed experiments in the Experimental section). This fumed catalytic treatment is a critical step for the successful coating of aluminum onto the substrate. After the catalytically treated substrate was dipped into the aluminum precursor composite solution, $\mathrm{AlH}_{3}\left\{\mathrm{O}\left(\mathrm{C}_{4} \mathrm{H}_{9}\right)_{2}\right\}$, prepared by a reaction of aluminum chloride $\left(\mathrm{AlCl}_{3}\right)$ with lithium aluminum hydride $\left(\mathrm{LiAlH}_{4}\right)$ in dibutyl ether $\left(\mathrm{O}\left(\mathrm{C}_{4} \mathrm{H}_{9}\right)_{2}\right)$, the Al-coated substrate appeared metallic gray in color. The detailed chemical mechanism of the decomposition of $\mathrm{AlH}_{3}\left\{\mathrm{O}\left(\mathrm{C}_{4} \mathrm{H}_{9}\right)_{2}\right\}$ for a highly conductive $\mathrm{Al}$ film on a catalytically treated substrate is illustrated in Fig. 1b. When the catalytically treated substrate was immersed into the $\mathrm{Al}$ precursor solution, $\mathrm{AlH}_{3}\left\{\mathrm{O}\left(\mathrm{C}_{4} \mathrm{H}_{9}\right)_{2}\right\}$, the catalyst coated onto the

\section{a}
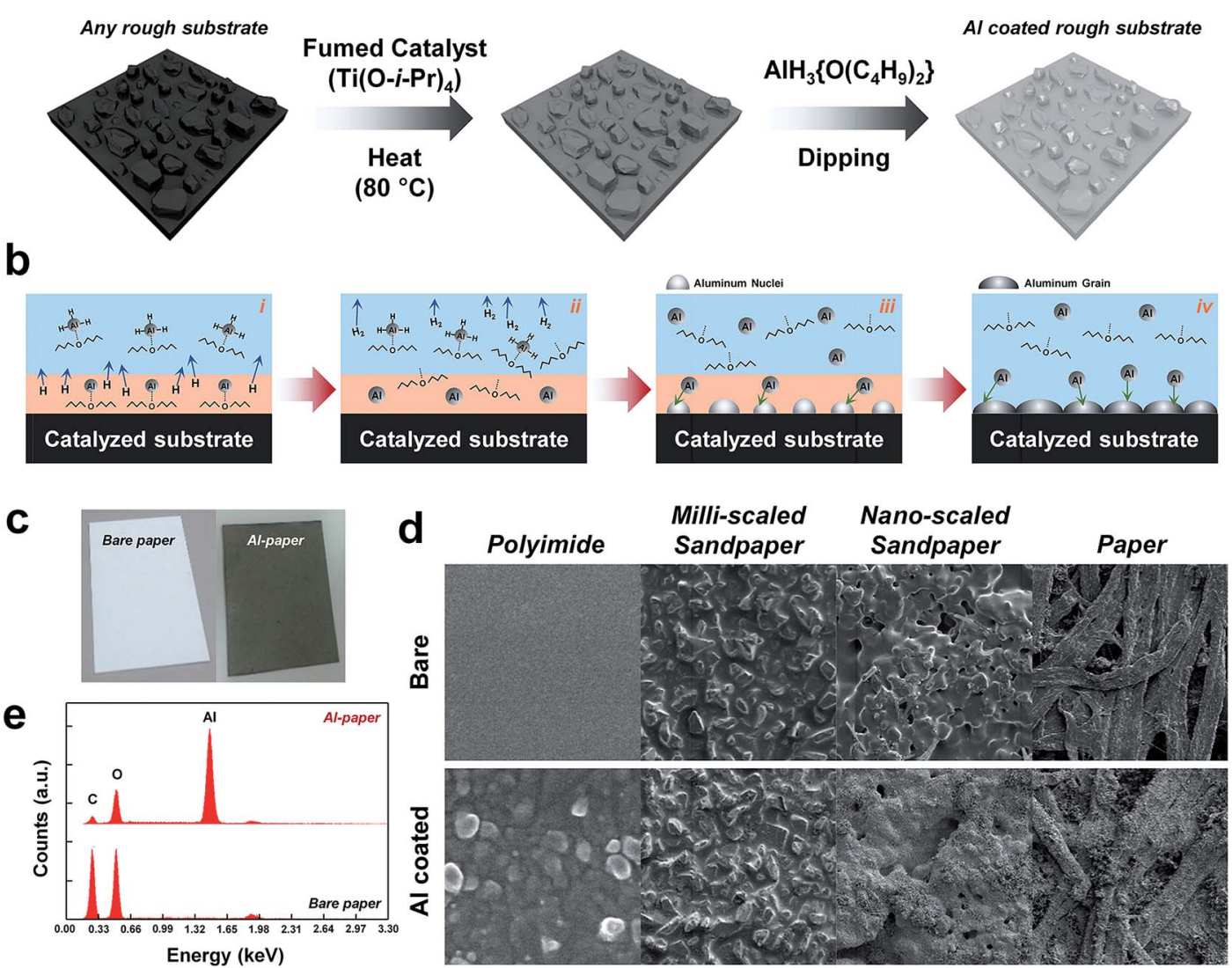

Fig. 1 Aluminum coated substrate by using solution process. (a) Schematics of the two-step fabrication procedures to depict the solutionprocessed aluminum coating (SC) method for the preparation of Al thin film with high electrical conductivity on a variety of large-sized substrates. (b) Detailed scheme of aluminum reduction from the $\mathrm{AlH}_{3}\left\{\mathrm{O}\left(\mathrm{C}_{4} \mathrm{H}_{9}\right)_{2}\right\}$ during the SC method. (c) Snapshots for (left) before and (right) after SC coating on a paper substrate. (d) Surface morphologies of four types of (top row) bare substrates and (bottom row) Al-coated substrates by use of scanning electron microscope (SEM). (e) Energy dispersive X-ray (EDAX) spectrums of (top) Al-coated paper and (bottom) bare paper. 
surface of the substrate activates the decomposition of $\mathrm{AlH}_{3}\{-$ $\left.\mathrm{O}\left(\mathrm{C}_{4} \mathrm{H}_{9}\right)_{2}\right\}$ into $\mathrm{Al}\left\{\mathrm{O}\left(\mathrm{C}_{4} \mathrm{H}_{9}\right)_{2}\right\}$ and $1.5 \mathrm{H}_{2}$, with the subsequent decomposition of $\mathrm{Al}\left\{\mathrm{O}\left(\mathrm{C}_{4} \mathrm{H}_{9}\right)_{2}\right\}$ into $\mathrm{Al}$ and $\left\{\mathrm{O}\left(\mathrm{C}_{4} \mathrm{H}_{9}\right)_{2}\right\}$. Finally, the nucleated $\mathrm{Al}$ grows large enough to cover the catalytically pretreated surface of the substrate (see the Fig. S1†).

The Al-coated paper substrate fabricated with the SC method is shown in Fig. 1c. Compared to uncoated paper, the Al-coated paper did not look shiny or metallic in color, instead appearing as gray in color. This arises from the optical scattering phenomenon, which occurs due to the inherently and randomly distributed rough morphology of the paper substrate. In contrast, the color of a flat surface substrate, polyimide (PI), when tested appeared shiny and metallic gray after being coating with aluminum using the SC method, as shown in Fig. S2. $\dagger$ The SC method can replace conventional physical vapor deposition processes such as metal evaporation and sputtering for metal film on various substrates, as it can be done at atmospheric pressure and at relatively low temperatures without concern over contamination originating from the substrate. This feature is especially attractive for electrodes used in TENGs. To investigate the surface morphologies of various substrates coated with Al using the SC method, a scanning electron microscope (SEM) was utilized. SEM images of various substrates including PI, common paper, and sandpaper, as shown in Fig. 1d. The upper row exhibits the surface morphologies of uncoated substrates, while the lower row exhibits those of the Al-coated substrates. It is clear that a range of substrates showing flat or rough surfaces even with a nano-tomicro scaled morphology can be conformally coated with the use of the SC method. ESI SEM images of other Al-coated sandpapers, including those with grit numbers of 150, 400, $800,1500,2 \mathrm{k}, 3 \mathrm{k}, 4 \mathrm{k}, 6 \mathrm{k}, 8 \mathrm{k}, 10 \mathrm{k}$, and $15 \mathrm{k}$ coated with the SC method, are shown in Fig. S3. $\dagger$ Further, a surface element analysis was conducted using energy dispersive X-ray (EDAX) spectroscopy to confirm that the Al film was coated onto the target substrates, as shown in Fig. 1e.

Fig. 2a illustrates schematics of the sandpaper TENG. A structure of the sandpaper TENG is composed of six parts. There are a top and a bottom acryl plate, which serve as an outer skeleton. Between them, four springs at each corner support the acryl plates. These springs also play a role in restoring the acryl plates to their original positions after the applied pressure is removed. The actual sandpaper TENG is composed of two electrodes, a top electrode ( $\mathrm{Au}$ ) and a contact electrode (Al). The polydimethylsiloxane (PDMS) elastomer is attached to top electrode (Au) film, which serve as a dielectric contact layer. The contact electrode is the sandpaper coated with Al by the SC method. The operation principle of the proposed TENG is depicted in Fig. 2b. This device generates electrical energy based on the actions of contact and separation. When the PDMS layer and the Al-coated sandpaper come into contact through an externally vertical force, electrons on the contact surface of the Al-coated sandpaper are locally transferred to the contact surface of the PDMS according to different triboelectric properties. This occurs through a physical phenomenon known as triboelectrification..$^{34}$ In general, at the moment of contact, the dielectric is negatively charged because it has more negative triboelectricity than the metal. Thus, current flows from the top
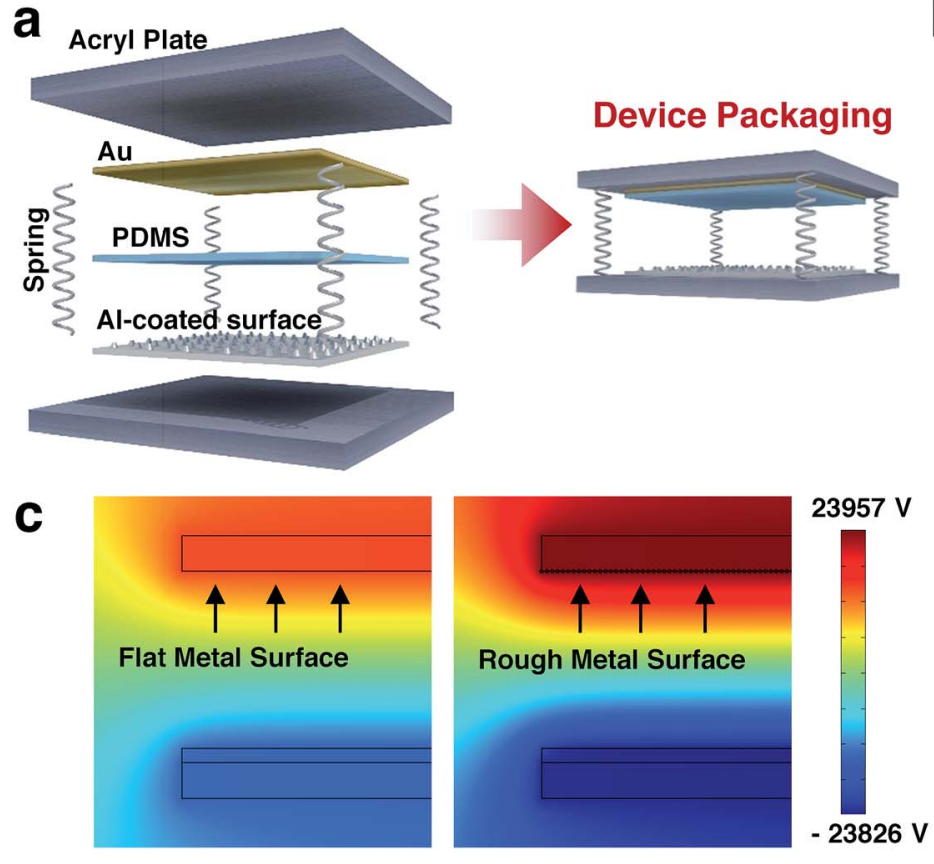

b

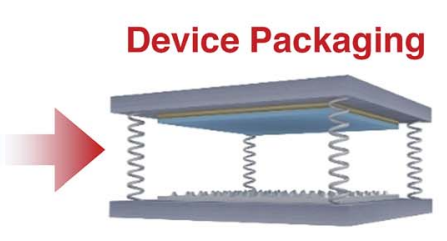

Fig. 2 Device structure and operation principle of the TENG. (a) Schematic illustration of the Al-coated sandpaper TENG. (b) Detailed illustration of the electrical output current flow via a load resistor in the contact-separation mode. (c) Simulation results showing the effect of a rough surface on the electric potential by from the COMSOL tool. Electric potential of (left) bare aluminum surface and (right) nano-to-micro structured aluminum surface. 
to the bottom electrode. In contrast, when the two surfaces are separated from each other by the restoring force arising from the four springs used in the device, the electrical potential between the two electrodes is changed due to the negatively charged PDMS layer by electrons which are transferred from the Al-coated sandpaper. Via the re-equilibrium potential, electrons in the Al-coated sandpaper move to the top Au electrode. Hence, current flows from the bottom to the top electrode. In this manner, the repetitive force causes in the sandpaper TENG a flow of alternating current between the two metal electrodes: top electrode $(\mathrm{Au})$ and contact electrode $(\mathrm{Al})$.

To visualize that the roughness of the metal surface affects the output performance of the TENG, a two-dimensional simulation of the mapping electric potential of flat metal and rough metal was performed using COMSOL Multiphysics, as shown in Fig. 2c. The simulation results indicate that the electric potential is significantly increased with the nano-tomicro structured aluminum. At the same external force, the surface morphology of the contact electrode (Al) results in an enlarged contact area between the two materials, generating an increased amount of triboelectric charge. In consequence, the rough metal surface shows four times the electrical potential as compared to the flat metal surface. It should be noted that the output performance of the sandpaper TENG is notably enhanced when using the SC method.

The overall electrical characteristics of TENGs consisting of different contact electrode substrates, in this case sandpapers with various grit numbers ranging from 150 to 15k and PI, PES,
PET, and A4-sized paper, are compared, as shown in Fig. 3. All output signals were measured under an identical external force of $70 \mathrm{~N}$ with a frequency of $3 \mathrm{~Hz}$, as provided from an electrodynamic shaker for precise quantitative analyses. The opencircuit voltage and short-circuit current of the sandpaper TENG showed values as high as $270 \mathrm{~V}$ and $77 \mu \mathrm{A}$, respectively, with a grit number of 15k, as shown in Fig. 3a and b. Moreover, forward and reverse-polarity switching tests of the open-circuit voltage of the TENG with a grit number of $15 \mathrm{k}$ were performed to confirm that this output signal is solely generated from the fabricated TENG, as shown in Fig. S4. $\dagger$ Further, the waveforms of various Al-coated substrates, such as sandpapers with grit numbers of $150,4 \mathrm{k}$, and $15 \mathrm{k}$ as well as common paper were measured, as shown in Fig. S5. $\dagger$ It is clear from the results that a more nano-scaled rough surface can generate a higher open-circuit voltage due to the increase in the surface charge density on the enlarged contact surface area. Considering that the common paper coated by the solution-processed aluminum intrinsically has its own roughness, it also shows a reasonably high open-circuit voltage. Therefore, this technique is considered as an 'appropriate technology', which is available at home.

The output power of the proposed TENG was characterized using an external load resistor. The peak value of the output voltage did not change with the load resistance at a low level, as shown in Fig. 3c. However, in the range of $10^{6} \Omega$ to $10^{8} \Omega$, it increases as the resistance increases. The value of the output current shows an opposite tendency compared to the value of the output voltage; i.e., it decreases as the load resistance
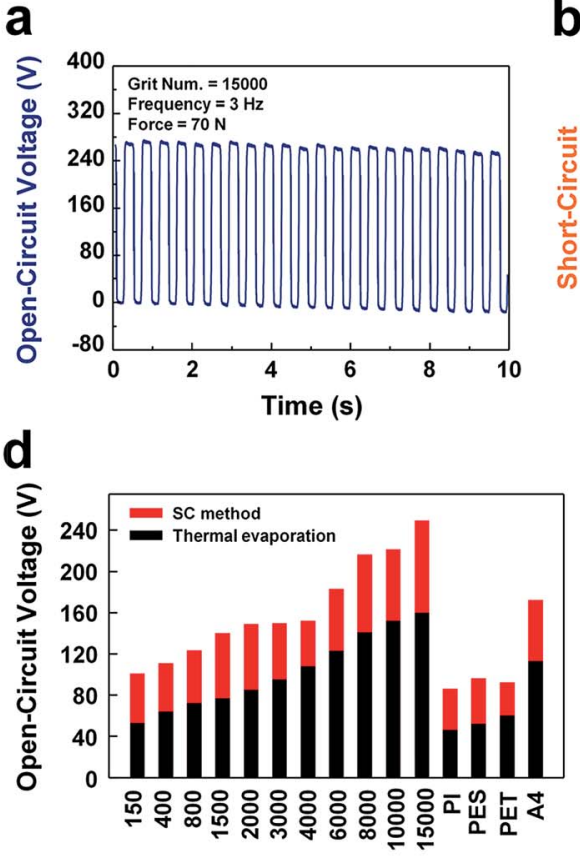

b

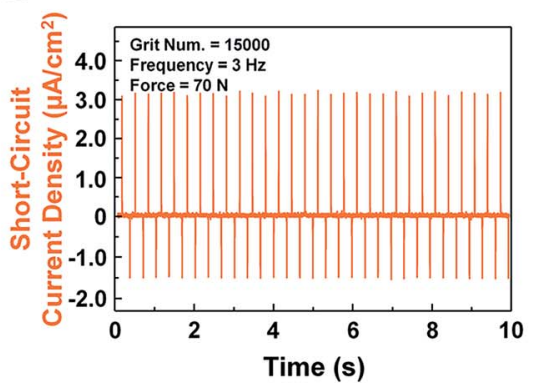

e

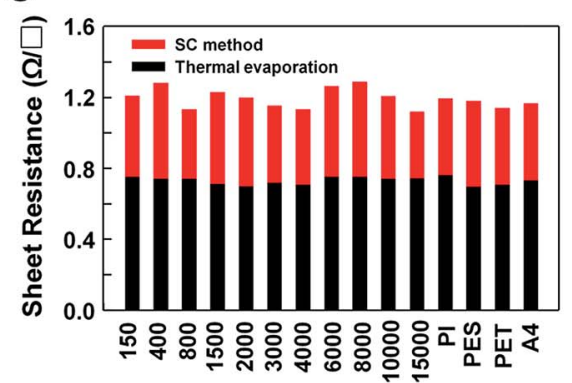

C

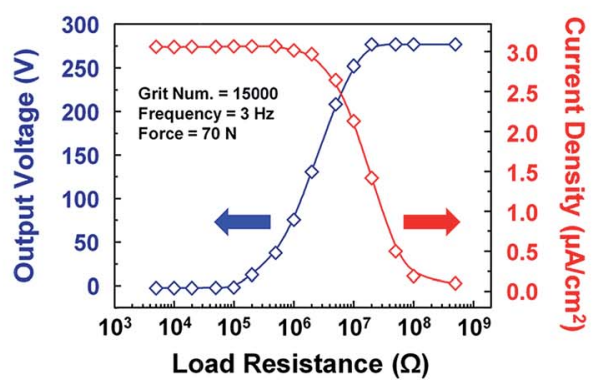

f

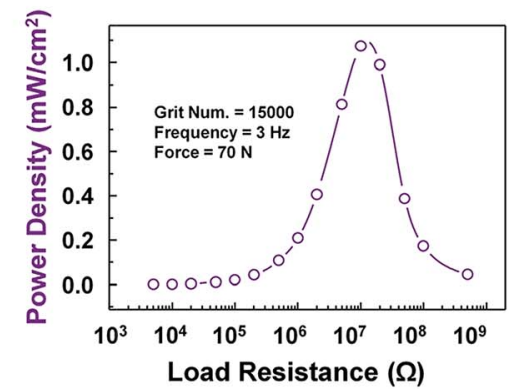

Fig. 3 Electrical characteristics of the fabricated sandpaper TENG. (a) Open-circuit voltage of the sandpaper TENG with a grit number of 15000 at a vibration frequency of $3 \mathrm{~Hz}$ under a force of $70 \mathrm{~N}$. (b) Short-circuit current of the sandpaper TENG with a grit number of 15000 at a vibration frequency of $3 \mathrm{~Hz}$ and a force of $70 \mathrm{~N}$. (c) Load resistance dependency of the open-circuit voltage and the short-circuit current with a grit number of 15 000. (d) Open-circuit voltage and (e) sheet resistance of various Al-coated substrates treated using (red) the SC method and (black) the thermal evaporation. (f) Load resistance dependency of the power density of the sandpaper TENG under the same experimental conditions of (c) according to the power density equation, $P=I^{2} R$ 
increases from $10^{5} \Omega$ to $10^{7} \Omega$ due to Ohm's law. Accordingly, the instantaneous peak power $\left(P_{2 \mathrm{D}}=I^{2} \times R\right)$ is maximized with a value of $1.1 \mathrm{~mW} \mathrm{~cm}^{-2}$ at a load resistance level of $10^{7} \Omega$. This value of the optimal load resistance implies that the internal resistance of the fabricated TENG is $10^{7} \Omega$. Hence, when the internal resistance equals the load resistance, the sandpaper TENG generates the maximum electrical power as shown in Fig. 3f.

Further, a variety of Al-coated substrates fabricated with the SC method exhibited high open-circuit voltages compared to those created with the conventional deposition process, thermal evaporation in this case, due to the fact that the Alcoated substrates fabricated with the SC method have more of a nano-to-micro surface morphology than those created by means of thermal evaporation. This is attributed to the good conformality of the solution-processed $\mathrm{Al}$, which retains the original form of the pre-existing nano-to-micro surface morphology on the substrates, as shown in Fig. 3d. On the other hand, the sheet resistance of the Al-coated substrates created via thermal evaporation is lower than those created with the SC method as shown in Fig. 3e. This tendency stems from the fact that the thermal evaporation is basically based on a vacuum process, which mitigates several contaminants on a target substrate. On the other hand, the SC method is based on solution process, which inevitably contains a few unreacted chemicals and by-products on the target substrate.

For reliable long-term operation of the TENG, a stringent requirement of the Al thin-film which covers the contact electrode is good mechanical durability, as the TENG inherently undergoes abrasion loss during the iterative contact and separation process. Fig. $4 \mathrm{a}$ and $\mathrm{b}$ show the data from the mechanical exfoliation test and from electrical endurance test, respectively. Scotch tape was used to investigate the $\mathrm{Al}$ adhesion to the substrate via a scratching and peeling-off process, serving as a metric by which to assess the durability of the device against damage. As shown in Fig. 4a, the Scotch tape tests were carried out by strongly attaching Scotch tape onto the Al-coated paper substrate created by the SC method and subsequently peeling it off. In the tests, none of the Al film samples were delaminated from any of the paper substrates. Furthermore, the electrical sheet resistance levels, as measured after the tape tests of all of the substrates, were scarcely changed from their initial values as shown in Fig. S6. $\dagger$ The results from the aforementioned tests support the contention that the adhesion of $\mathrm{Al}$ to the paper substrates was so strong that the Al thin-film on the substrates created by the SC method did not undergo delamination, which in turn decreases the electrical conductivity. Such strong binding of Al to a paper substrate presumably stemmed from the Al precursor solution smeared into the paper, causing the $\mathrm{Al}$ thin film to be created at both the outer and inner sides of the paper substrate. To investigate the actual mechanical and electrical stability of the proposed TENG, it was intentionally exposed to the harsh stress condition of non-stop operation for nine days, which corresponds to $3.9 \times 10^{6}$ cycles with a vibrating frequency of $5 \mathrm{~Hz}$ as shown in Fig. $4 \mathrm{~b}$. The amount of output voltage generated was held nearly constant without

\section{a}

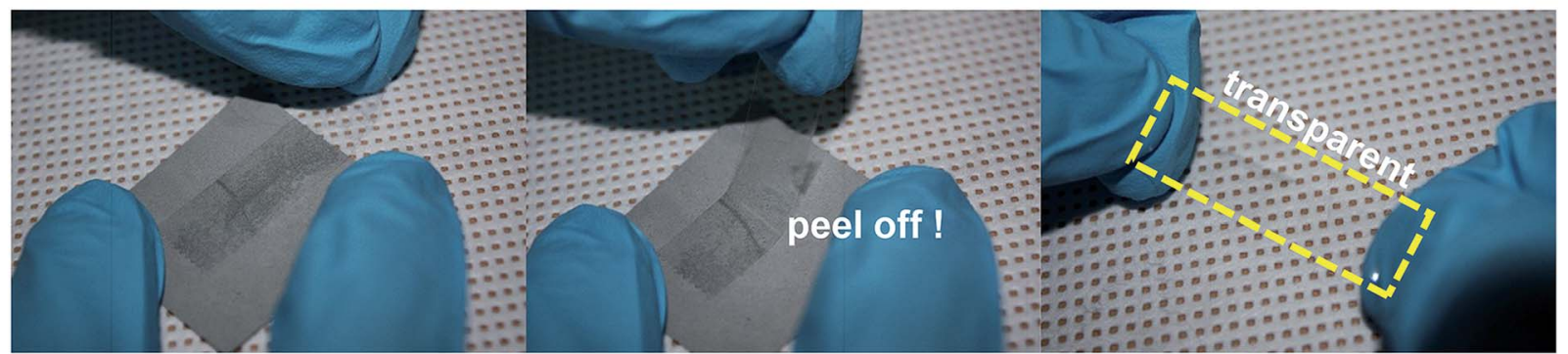

b

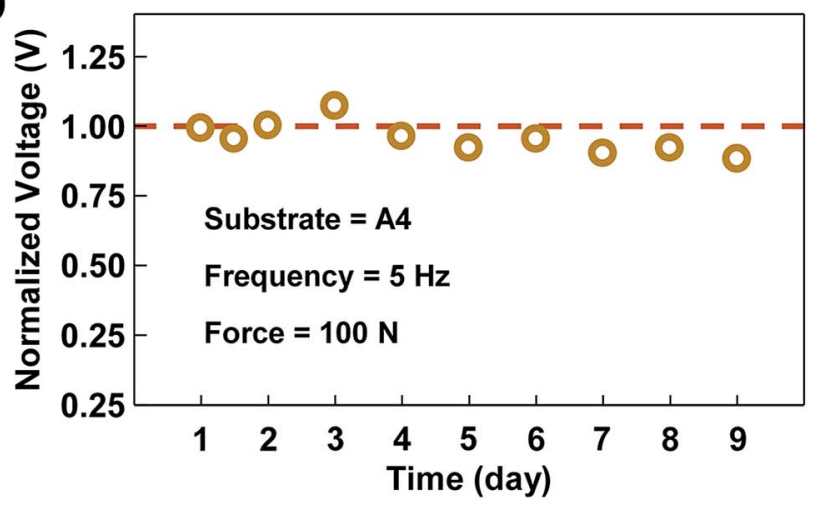

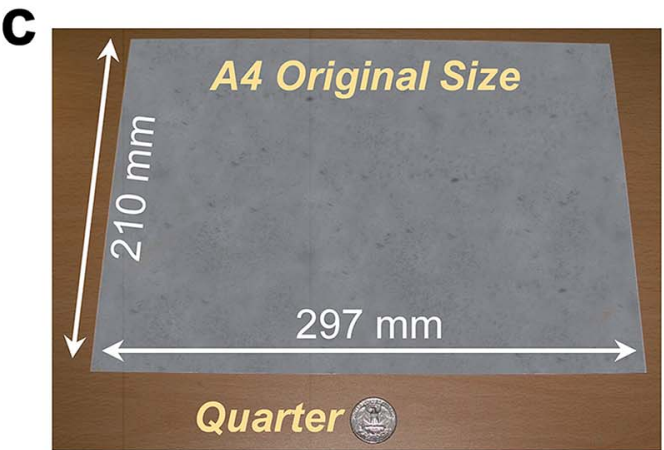

Fig. 4 Endurance and feasibility of the large-scale fabrication. (a) Scotch tape adhesion test for the Al-coated paper substrate using the SC method. (b) Endurance test of the fabricated sandpaper TENG by using Al-coated paper for more than 9 days under a vertical force of $100 \mathrm{~N}$ at a vibration frequency of $5 \mathrm{~Hz}$. (c) Digital snapshot of the large A4-sized Al-coated paper. 
a
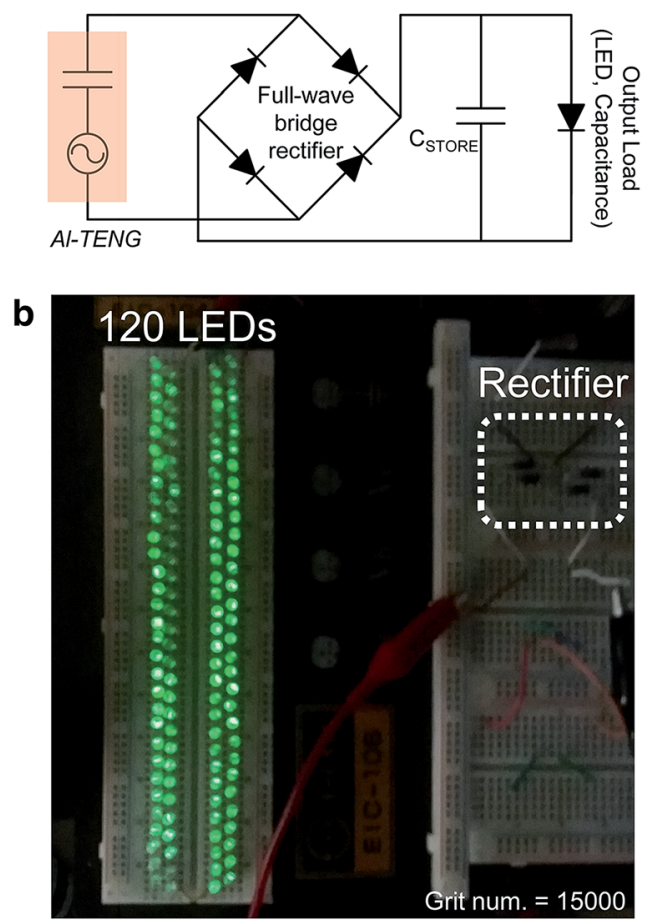

C

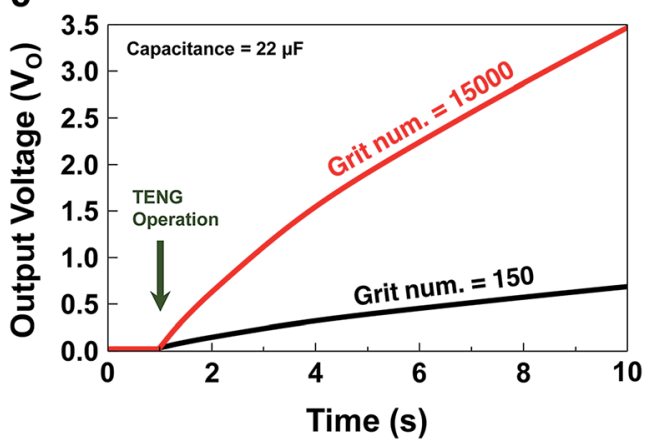

Fig. 5 Charging and powering electronics. (a) Circuit diagram of a fullwave bridge rectifier. (b) Digital snapshot to show the simultaneously powering of 120 green LEDs via a serial connection. (c) Charging behavior of a commercial capacitor $(22 \mu \mathrm{F})$ connected to the Alcoated sandpaper with the grit numbers of (red) 15k and (black) 150 for $10 \mathrm{~s}$.

considerable degradation, even after $3.9 \times 10^{6}$ cycles of vibration. The sandpaper TENG showed very reliable durability due to the unique feature of the micro-sized powder, which does not undergo abrasion. This is not the case if a solid plate is used as the triboelectric electrode. This excellent durability reveals that the sandpaper TENG is advantageous as a sustainable power source. In addition to providing good electrical and mechanical durability, the suggested SC method has an additional advantage. With this method, $\mathrm{Al}$ coatings on the target substrates are not restricted by the size of the substrate because this method is based on a solution process. In previous research, physical vapor deposition (PVD) methods such as thermal or electron beam evaporation and a sputtering method were used to deposit Al on a substrate.
However, these methods are not always possible with a largesized substrate owing to the limited size of the vacuum chamber typically used. However, when using the SC method, it becomes possible to coat $\mathrm{Al}$ onto a large-area substrate because the formation of the thin film relies not on physical deposition under an ambient vacuum but on only chemical dipping into the Al precursor solution. Fig. 4c shows a piece of Al-coated A4 paper $(210 \mathrm{~mm} \times 297 \mathrm{~mm})$ fabricated using the SC method. These images demonstrate that the Al coating is homogeneously formed, judging from its metallic gray color throughout the area of the paper.

To demonstrate the capability of the fabricated TENG as a power source for small electronic devices, commercial LEDs and capacitors were connected to the sandpaper TENG each other. To deliver the rectified output signal to the small electronics, a full-wave bridge rectifier circuit was used as shown in Fig. 5a. When the sandpaper TENG was vibrated at a frequency of $3 \mathrm{~Hz}, 120$ serially connected green LEDs were lit instantly. A digital snapshot of the turned-on LEDs is shown in Fig. 5b. It is noteworthy that the illuminated LEDs driven by the sandpaper TENG were clearly visible even at background light illumination of 146 lux (see the ESI Video S1 $\dagger$ ). To demonstrate the charging behavior by the fabricated TENG, a $22 \mu \mathrm{F}$ capacitor was utilized for electric energy storage. The charging curves of the capacitor charged by the generated power from two sandpaper TENGs with grit numbers of 150 and 15k are shown in Fig. 5c. The TENG with the grit number of $15 \mathrm{k}$ produces output voltage $\left(V_{\mathrm{O}}\right)$ of $3.5 \mathrm{~V}$ after charging the capacitor at $22 \mu \mathrm{F}$ for $10 \mathrm{~s}$. In contrast, that with the grit number of 150 generates $V_{\mathrm{O}}$ of $0.5 \mathrm{~V}$ after charging the same capacitor for $10 \mathrm{~s}$. This tendency is attributed to the fact that the sandpaper with the larger grit number has a greater nano-to-micro surface morphology. Thus it is concluded that sandpaper with a larger grit number has more triboelectricity, as predicted. The capabilities of the sandpaper TENG can be ready quickly for commercialization at a low cost and with 'appropriate technology'.

\section{Conclusions}

In summary, a novel triboelectric nanogenerator composed of a contact electrode in the form of sandpaper conformally coated by solution-processed aluminum was developed. As a control group, various substrates, including common paper, PI, PES, and PET, were also prepared using the same solution-processed aluminum. A maximum voltage of $270 \mathrm{~V}$, maximum current of $77 \mu \mathrm{A}$, and a maximum power density of $1.1 \mathrm{~mW} \mathrm{~cm}^{-2}$ were achieved from sandpaper with a grit number of $15 \mathrm{k}$. It was shown that a greater grit number produced more power. In addition, the sandpaper TENG showed excellent durability against mechanical friction. Moreover, it is capable of serving as an electrical power supplier for the wireless sensor networks (WSNs) used in the era of the Internet of Things (IoT) due to the low-cost fabrication process facilitated by the use of atmospheric pressure, a large-sized substrate, and inherently pre-existing nano-tomicro morphology substrate. 


\section{Experimental}

Preparation of the aluminum precursor solution of $\mathrm{AlH}_{3}\left\{\mathrm{O}\left(\mathrm{C}_{4} \mathrm{H}_{9}\right)_{2}\right\}$

The Al precursor solution was prepared by an ethereal reaction of aluminum chloride $\left(\mathrm{AlCl}_{3}\right)$ with lithium aluminum hydride $\left(\mathrm{LiAlH}_{4}\right)$ in dibutyl ether $\left(\mathrm{O}\left(\mathrm{C}_{4} \mathrm{H}_{9}\right)_{2}\right) \cdot \mathrm{AlCl}_{3}, \mathrm{LiAlH}_{4}$, and $\mathrm{O}\left(\mathrm{C}_{4} \mathrm{H}_{9}\right)_{2}$ were purchased from Sigma Aldrich and used as received. $\mathrm{AlCl}_{3}$ was used as a precursor for preparing the $\mathrm{Al}$ precursor solution, while $\mathrm{LiAlH}_{4}$ was used as both a precursor and a reduction agent. Dibutyl ether was used as the solvent for the reactions of both chemicals. For the reaction, $20 \mathrm{mM}$ of $\mathrm{AlCl}_{3}$ and $60 \mathrm{mM}$ of $\mathrm{LiAlH}_{4}$ were added to $50 \mathrm{~mL}$ of dibutyl ether. The mixed solution was heated at approximately $75{ }^{\circ} \mathrm{C}$ while stirring for $1 \mathrm{~h}$. To remove any byproduct of $\mathrm{LiCl}$ and precursors which were not fully reacted, the resulting gray slurry was then filtered and then discarded. The filtered clear solution was used as an $\mathrm{Al}$ precursor solution of $\mathrm{AlH}_{3}\left\{\mathrm{O}\left(\mathrm{C}_{4} \mathrm{H}_{9}\right)_{2}\right\}$.

\section{Catalytic treatment of substrate}

Titanium isopropoxide $\left(\mathrm{Ti}(\mathrm{O}-\mathrm{i}-\mathrm{Pr})_{4}\right)$ is a catalyst popularly used for the chemical preparation of pure $\mathrm{Al}$, as it is extremely effective for the dehydrogenation of $\mathrm{AlH}_{3}$. Hence, we utilized $\mathrm{Ti}(\mathrm{O}-\mathrm{i}-\mathrm{Pr})_{4}$ for the catalytic treatment of the target substrate (sandpapers, paper, polyimide). The catalytic treatment of the substrate was conducted by exposing the substrate to the titanium isopropoxide ( $\left.\mathrm{Ti}(\mathrm{O}-\mathrm{i}-\mathrm{Pr})_{4}\right)$ in a fumed state for $10 \mathrm{~min}$. This catalytic treatment was carried out in a glove box filled with Ar gas, with the fumed $\mathrm{Ti}(\mathrm{O}-\mathrm{i}-\mathrm{Pr})_{4}$ not easily oxidized to $\mathrm{TiO}_{2}$. The target substrates was placed on the surface of a hotplate which was preheated to $110^{\circ} \mathrm{C}$, and approximately $10 \mu \mathrm{L}$ of the catalyst was dropped onto the surface of the heated plate together with the substrate. Since the catalyst dropped onto the plate preheated at $110{ }^{\circ} \mathrm{C}$ instantly changed into fumes, the surface of the substrate was exposed to the fumed catalyst. This treated substrate was then dried under inert Ar or $\mathrm{N}_{2}$ gas.

\section{Characterization}

Morphology of various Al-coated substrates treated by the SC method was confirmed using a scanning electron microscope (SEM, Magellan 400) at an operation voltage of $10 \mathrm{kV}$. Chemical compositions of the Al-coated substrates were obtained by means of energy dispersive X-ray spectroscopy (EDAX, AMETEK).

\section{Electrical measurement}

An electrodynamic shaker (Labworks Inc., ET-140) converts electrical signals into the mechanical 1D movement and can therefore be used to apply vertical force to the TENG. The output voltage and current from the fabricated TENG were measured by an electrometer (Keithley, 6514).

\section{Acknowledgements}

This work was partially supported by Open Innovation Lab Project from the National NanoFab Center (NNFC) and the End
Run Project funded by the Ministry of Science, ICT \& Future Planning. It was also supported by the Center for Integrated Smart Sensors funded by the Ministry of Science, ICT \& Future Planning as part of the Global Frontier Project (CISS-20110031848).

\section{Notes and references}

1 Z. L. Wang, G. Zhu, Y. Yang, S. Wang and C. Pan, Mater. Today, 2012, 15, 532.

2 Z. L. Wang and J. Song, Science, 2006, 312, 242.

3 J. H. Jung, M. Lee, J.-I. Hong, Y. Ding, C.-Y. Chen, L.-J. Chou and Z. L. Wang, ACS Nano, 2011, 5, 10041.

4 Y. Mao, P. Zhao, G. McConohy, H. Yang, Y. Tong and X. Wang, Adv. Energy Mater., 2014, 4, 1301624.

5 C. R. Saha, T. O'Donnell, H. Loder, S. Beeby and J. Tudor, IEEE Trans. Magn., 2006, 42, 3509.

6 C. R. Saha, T. O'Donnell, N. Wang and P. McCloskey, Sens. Actuators, A, 2008, 147, 248.

7 B. Yang, C. Lee, W. Xiang, J. Xie, J. H. He, R. K. Kotlanka, S. P. Low and H. Feng, J. Micromech. Microeng., 2009, 19, 035001.

8 Y. Naruse, N. Matsubara, K. Mabuchi, M. Izumi and S. Suzuki, J. Micromech. Microeng., 2009, 19, 094002.

9 E. O. Torres and G. A. Rincón-Mora, IEEE Transactions on Circuits and Systems I: Regular Papers, 2009, 56, 1938.

10 E. O. Torres and G. A. Rincón-Mora, IEEE J. Solid-State Circuits, 2010, 45, 483.

11 F.-R. Fan, Z.-Q. Tian and Z. L. Wang, Nano Energy, 2012, 1, 328.

12 Z. L. Wang, Faraday Discuss., 2014, 176, 447.

13 K. Y. Lee, M. K. Gupta and S.-W. Kim, Nano Energy, 2015, 14, 139.

14 Q. Liang, X. Yan, X. Liao, S. Cao, X. Zheng, H. Si, S. Lu and Y. Zhang, Nano Energy, 2015, 16, 329.

15 Q. Liang, X. Yan, Y. Gu, K. Zhang, M. Liang, S. Lu, X. Zheng and Y. Zhang, Sci. Rep., 2015, 5, 9080.

16 Q. Liang, Z. Zhanga, X. Yan, Y. Gu, Y. Zhao, G. Zhang, S. Lu, Q. Liao and Y. Zhang, Nano Energy, 2015, 14, 209.

17 W. Tang, C. Zhang, C. B. Han and Z. L. Wang, Adv. Funct. Mater., 2014, 24, 6684.

18 L. Lin, Y. Xie, S. Niu, S. Wang, P. K. Yang and Z. L. Wang, ACS Nano, 2015, 9, 922.

19 D. Kim, S.-J. Park, S.-B. Jeon, M.-L. Seol and Y.-K. Choi, Adv. Electron. Mater., 2016, 2, 1500331.

20 D. Kim, S.-B. Jeon, J. Y. Kim, M.-L. Seol, S. O. Kim and Y.-K. Choi, Nano Energy, 2015, 12, 331.

21 Z. H. Lin, G. Cheng, W. Wu, K. C. Pradel and Z. L. Wang, ACS Nano, 2014, 8, 6440.

22 H. Zhang, Y. Yang, X. Zhong, Y. Su, Y. Zhou, C. Hu and Z. L. Wang, ACS Nano, 2014, 8, 680.

23 S. Kim, M. K. Gupta, K. Y. Lee, A. Sohn, T. Y. Kim, K.-S. Shin, D. Kim, S. K. Kim, K. H. Lee, H.-J. Shin, D.-W. Kim and S.-W. Kim, Adv. Mater., 2014, 26, 3918.

24 D. Kim, Y. Oh, B.-W. Hwang, S.-B. Jeon, S.-J. Park and Y.-K. Choi, ACS Nano, 2016, 10, 1017. 
25 K. Y. Lee, J. Chun, J.-H. Lee, K. N. Kim, N.-R. Kang, J.-Y. Kim, M. H. Kim, K.-S. Shin, M. K. Gupta, J. M. Baik and S.-W. Kim, Adv. Mater., 2014, 26, 5037.

26 F. Yi, X. Wang, S. Niu, S. Li, Y. Yin, K. Dai, G. Zhang, L. Lin, Z. Wen, H. Guo, J. Wang, M.-H. Yeh, Y. Zi, Q. Liao, Z. You, Y. Zhang and Z. L. Wang, Sci. Adv., 2016, 2, e1501624.

27 J. Wang, S. Li, F. Yi, Y. Zi, J. Lin, X. Wang, Y. Xu and Z. L. Wang, Nat. Commun., 2016, 7, 12744.

28 F. Yi, J. Wang, X. Wang, S. Niu, S. Li, Q. Liao, Y. Xu, Z. You, Y. Zhang and Z. L. Wang, ACS Nano, 2016, 10, 6519.
29 X.-S. Zhang, M.-D. Han, R.-X. Wang, F.-Y. Zhu, Z.-H. Li, W. Wang and H.-X. Zhang, Nano Lett., 2013, 13, 1168.

30 M. Chen, X. Li, L. Lin, W. Du, X. Han, J. Zhu, C. Pan and Z. L. Wang, Adv. Funct. Mater., 2014, 24, 5059.

31 J. Yang, J. Chen, Y. Liu, W. Yang, Y. Su and Z. L. Wang, ACS Nano, 2014, 8, 2649.

32 Y. Yang, G. Zhu, H. Zhang, J. Chen, X. Zhong, Z. H. Lin, Y. Su, P. Bai, X. Wen and Z. L. Wang, ACS Nano, 2013, 7, 9461.

33 H. M. Lee, S.-Y. Choi, A. Jung and S. H. Ko, Angew. Chem., Int. Ed., 2013, 52, 7718.

34 P. E. Shaw, Proc. R. Soc. London, Ser. A, 1917, 94, 16. 